Seyla Benhabib

\section{Las reivindicaciones de la cultura. Igualdad y diversidad en la era global}

(Buenos Aires, Katz, 2006)

Las «luchas por el reconocimiento» (Taylor, Fraser, Honneth), los «movimientos por la identidad/diferencia» (Young, Connolly), los «movimientos por los derechos culturales y la ciudadanía multicultural» (Kymlicka), dan cuenta de un nuevo imaginario característico de nuestro tiempo, en el que el tema de la identidad cultural ocupa el primer plano del discurso político. Es cierto que la cultura siempre ha sido un indicador de la diferencia social; lo que resulta novedoso es que los grupos que actualmente se constituyen en torno a dichos indicadores identitarios exigen un reconocimiento legal y la intervención de las instituciones estatales para preservar y proteger sus diferencias culturales.

Éste es el marco en el que se sitúa la obra de Seyla Benhabib sobre las reivindicaciones de la cultura. En ella examina los desafíos que la coexistencia de estos movimientos en el mismo espacio político-temporal plantea a la teoría y la práctica de las democracias liberales. Pretende dar respuesta a las tensiones que las políticas de la identidad plantean a la democracia liberal a partir de una combinación de los postulados de la Escuela de Frankfurt con la teoría feminista, tratando de encontrar un punto intermedio entre el universalismo ético habermasiano y el particularismo culturalista.
Benhabib sostiene que la respuesta a esa «extraña multiplicidad» de nuestro tiempo (Tully, 1995) ha partido con frecuencia de «la reificación demasiado expeditiva de identidades grupales dadas, el fracaso para cuestionar el significado de la identidad cultural y el abandono de estas temáticas por parte de la literatura sociológica e histórica, dominadas por el constructivismo metodológico. El resultado de este normativismo prematuro es la implementación de políticas improvisadas que corren el riesgo de solidificar las diferencias ya existentes entre los grupos» (Benhabib, 2006: 9). De la consideración de las culturas como totalidades estáticas y cerradas se derivan una serie de problemas para la teoría y la práctica democráticas; entre otros, la dificultad para conciliar el respeto a las culturas con la autonomía individual, la compatibilidad entre universalismo y particularismo o la posibilidad del diálogo intercultural.

Para salvar estos problemas, Benhabib propone «un modelo democrático deliberativo que permita la máxima controversia cultural dentro de la esfera pública» (Benhabib, 2006: 9). Es decir, frente al esencialismo que caracteriza a muchos de los planteamientos de las políticas de la identidad en sus diferentes versiones, apuesta por una idea de las culturas como «prácticas humanas complejas de significación y representación, de organización y atribución, divididas internamente por relatos en conflicto. Las culturas se crean a partir de diálogos complejos con otras culturas» (Benhabib, 2006: 10). La complejidad interna y el carácter controvertido es algo inherente a toda cultura.

Ésta es la tesis central que plantea en los dos primeros capítulos. En ellos desarrolla su idea 
de la cultura, las identidades humanas y esboza el enfoque de los diálogos culturales complejos. Una gran parte del debate actual sobre las reivindicaciones culturales, mantiene la autora, está dominado por una «sociología reduccionista de la cultura», que considera que las culturas son entidades cuyos límites pueden identificarse claramente, que se corresponden con los grupos de población, dentro de los cuales es posible describir la cultura de una forma no controvertida, y que la existencia de más de una cultura dentro de un grupo humano, o la no coincidencia exacta entre grupos y culturas, no plantea problemas significativos para las correspondientes políticas. Esto trae consigo innumerables problemas respecto a la forma de pensar la manera de promover el pluralismo y la diversidad humana. Frente al esencialismo cultural, propone una «visión narrativa de las acciones y la cultura» (Benhabib, 2006: 29), que distinga entre el observador social y el agente social. «Cualquier visión de las culturas como totalidades claramente definibles es una visión desde fuera que genera coherencia con el propósito de comprender y controlar. Por el contrario, los participantes de la cultura experimentan sus tradiciones, historias, rituales y símbolos, herramientas y condiciones materiales de vida a través de relatos narrativos compartidos, aunque también controvertidos y factibles de ser rebatidos» (Benhabib, 2006: 29). El constructivismo sociológico que está en la base de la consideración objetiva de las culturas conduce a un «multiculturalismo mosaico», que llevaría a absolutizar la defensa de las culturas por encima de la justicia y la libertad individuales y haría difícil el diálogo entre las culturas. Frente a ese multiculturalismo fuerte deberíamos considerar las culturas humanas como constantes creaciones, recreaciones y negociaciones de fronteras imaginarias entre «nosotros y el/los otro(s)» (Benhabib, 2006: 33).

La misma visión estática y cerrada de las culturas subyace a la pregunta planteada en el capítulo tercero: « ¿Es etnocéntrico el universalismo?». Pregunta que revela un temor que ha desvelado a Occidente desde la conquista de América y que responde a una consideración del carácter homogéneo de la identidad occidental, de su radical distinción con los sistemas de valores y formas de vida de otras civilizaciones, lo cual nos permitiría trazar claramente la frontera entre «nosotros» y «los otros». Frente a ello, Benhabib propone que «en sí mismas, ni las culturas ni las sociedades son holísticas, sino que son sistemas de acción y significados polivocales, descentrados y fracturados, que abarcan varios niveles» (Benhabib, 2006: 61).

Los capítulos tercero y cuarto tratan sobre la política de la identidad y de la diferencia en un contexto global y algunas de las tensiones que ésta plantea con los principios de la democracia liberal y, sobre todo, con la autonomía individual. En ellos analiza el cambio de paradigma de la redistribución al reconocimiento en la política actual, a la luz del examen crítico de tres teorías contemporáneas sobre el reconocimiento, las de Charles Taylor, Will Kymlicka y Nancy Fraser. Concretamente, critica la relación que establece Taylor entre las demandas de reconocimiento en el plano individual y el nivel colectivo. «Las reivindicaciones individuales de autoexpresión auténtica no tienen por qué ir de la mano de las aspiraciones colectivas al reconocimiento cultural»(Benhabib, 2006: 99), 
señala la autora. En ocasiones, incluso, puede existir una contradicción entre ambos niveles. En el planteamiento de Taylor subyace un error teórico que consiste en equiparar las reivindicaciones individuales y colectivas. Además, esta equiparación es peligrosa a nivel político, puesto que lleva a subordinar la autonomía individual a los movimientos por el reconocimiento de la identidad colectiva. Taylor debería admitir la posibilidad de que los movimientos colectivos por la afirmación de los derechos grupales entren en conflicto con las reivindicaciones individuales de autonomía.

A este problema trata de hacer frente Kymlicka con su distinción entre «restricciones internas» $\mathrm{y}$ «protecciones externas», las primeras relativas a las reivindicaciones de un grupo frente a sus miembros, las segundas a las que los miembros de un grupo dirigen contra la sociedad en general; las primeras destinadas a salvaguardar la autonomía indvidual frente al grupo, las segundas a la protección de las culturas. Sin embargo, Kymlicka lleva a cabo una reificación ilegítima de las identidades nacionales y etnoculturales al equiparar el concepto de cultura con las formas institucionales de cultura, lo que él Ilama una «cultura societal» —una cultura que se manifiesta en las instituciones públicas, en la educación, la administración, los medios de comunicación-, y a partir de ahí considera que únicamente están justificadas las demandas de derechos diferenciados llevadas a cabo por las minorías nacionales, pero no las de los grupos etnoculturales que se van formando en un país, por ejemplo, como consecuencia de la inmigración. Por ello, señala Benhabib, su planteamiento adolece también del mismo reduccionismo y objetivismo en la consideración de las culturas como algo estático y perfectamente delimitable a partir de criterios objetivistas, como la concentración territorial o la viabilidad de una cultura societaria. Pero no tiene en cuenta las construcciones dinámicas de la identidad, es decir, el hecho de que la diferencia entre minorías nacionales -aquellas que están concentradas en un territorio y dan lugar a Estados multinacionales-y grupos etnoculturales - los grupos que se van formando a partir de la inmigración y que forman Estados poliétnicos— «no es estática sino dinámica, y ella sola no basta para diferenciar entre las reivindicaciones por el reconocimiento y las aspiraciones de agrupaciones humanas diferentes» (Benhabib, 2006: 117). Es decir, el tipo de grupo al que uno considera que pertenece puede cambiar en el transcurso de la propia lucha política, de forma que un grupo étnico empiece a considerarse como una minoría nacional. Quizá para discriminar hasta qué punto unas demandas están justificadas o no habría que recurrir no tanto a criterios objetivistas, como la concentración territorial, que nos dicen poco acerca de la compatibilidad o no de las reivindicaciones con los principios liberales, sino a lo que los actores políticos están diciendo sobre sus propios objetivos y cómo están actuando para llevarlos a cabo. Pero los propios argumentos de Kymlicka para reconciliar el liberalismo con los derechos de las culturas se basan más en premisas multiculturalistas que en evaluaciones políticas de los movimientos reivindicativos y sus demandas; por eso no está justificada la discriminación de un determinado tipo de demandas. «Si la cultura es valiosa desde el punto de vista del liberalismo político porque permite un espectro significativo de elecciones en el manejo de 
nuestras vidas y porque configura los parámetros dentro de los cuales creamos un plan de vida en primer lugar, entonces, objetivamente, no existe ninguna base para que el teórico privilegie las culturas nacionales por encima de las inmigrantes, o las culturas de grupos religiosos por encima de las de los movimientos sociales» (Benhabib, 2006: 121). No cabe recurrir a argumentos externos al carácter y al significado de las propias reivindicaciones llevadas a cabo por los grupos y a su compatibilidad con los principios liberales; de manera especial, con la autonomía individual.

En contraste con las tesis objetivistas de Taylor y Kymlicka, la postura de Nancy Fraser no conduce necesariamente al esencialismo cultural, ya que para Fraser la política del reconocimiento no puede equipararse con la política identitaria. La reafirmación del derecho de los grupos a definir los contenidos y los límites de la propia identidad no es la única manera de hacer justicia a las reivindicaciones por el reconocimiento; también cabe responder mediante el cambio en los patrones culturales de interpretación, comunicación y representación que lleve a una redefinición del estatus cultural de los grupos que sufren una falta de reconocimiento y que deriven en una mejora de su situación socioeconómica.

En esta misma línea, el capítulo cuarto, sobre «El multiculturalismo y la ciudadanía de género», discute los dilemas planteados por la dificultad para conciliar la preservación de las identidades culturales con los derechos individuales, a partir del estudio de tres casos: la defensa jurídica basada en argumentos culturales, el impacto del código familiar privado en las vidas de las mujeres musulmanas en la India y el debate sobre el pañuelo islámico en Francia. Es posible superar la posible contradicción entre la defensa de la cultura y la protección de la autonomía de las mujeres abandonando una concepción reificada y estática de las culturas y mediante un modelo de democracia deliberativa que parta de la confianza en la capacidad de los actores para renegociar sus propios relatos de identidad y de diferencia a través de los encuentros multiculturales en la sociedad civil democrática. «Una política multicultural democrática y deliberativa no relega a las mujeres, niños y niñas a sus comunidades de origen en contra de su voluntad, sino que alienta el desarrollo de su agencia autónoma frente a las identidades que les son asignadas» (Benhabib, 2006: 151).

Esta propuesta de democracia deliberativa es la que va a desarrollar en el capítulo quinto. Lo que ella denomina el enfoque de «doble vía», que remarca la importancia tanto de las instituciones legislativas, políticas y judiciales como de las asociaciones de ciudadanos, los grupos de interés y los movimientos sociales en la esfera pública, frente a otros modelos como el «consenso superpuesto» de Rawls, el «igualitarismo liberal» de Brian Barry o las «jurisdicciones multiculturales» de Ayelet Shachar. Las propuestas de estos autores tienden a concentrarse en la esfera pública oficial; en cambio, el enfoque de la doble vía, que da una mayor importancia a la sociedad civil, resulta más adecuado para resolver las cuestiones y los conflictos multiculturales, puesto que abre un espacio de diálogo para comprender los procesos de resignificación de las prácticas culturales. Por ejemplo, si se hubiera escuchado lo 
que tenían que decir las niñas que acudían a clase con el velo islámico en Francia se habría entendido que el significado del pañuelo estaba cambiando, de ser un acto religioso a convertirse en un acto de desafío cultural y creciente politización, de expresión de una situación de malestar social y de crisis de identidad. Algo que no parece que afecte directamente a la laicidad del Estado y que vuelve a encerrar a esas chicas dentro de los muros de sentido de los que tal vez están tratando de escapar mediante la educación.

Para terminar analiza las transformaciones de la ciudadanía en Europa como consecuencia de un doble proceso de unificación, por un lado, y las fuerzas del multiculturalismo, la inmigración y los separatismos culturales, por otro. Señala cómo la situación de los residentes no ciudadanos cuestiona la interdependencia entre nacionalidad y ciudadanía que ha estado en la base del desarrollo del Estado nacional moderno. Apunta la autora que estamos asistiendo a un "efecto de desagregación» de los distintos componentes de la ciudadanía: la identidad colectiva y los derechos políticos y sociales, en el sentido de que no hay una coincidencia exacta entre quienes comparten la identidad cultural que históricamente ha dotado de legitimidad al Estado nacional, quienes gozan de derechos políticos y quienes se benefician de las prestaciones del Estado de bienestar. Las teorías clásicas sobre la ciudadanía se han apoyado normalmente en la ficción de una "sociedad cerrada", en el "nacionalismo metodológico» (Smith, 1979). Pero la aceleración de la movilidad humana a través de las fronteras de los Estados nacionales obliga a replantearse la línea que separa a los incluidos de los excluidos, es decir, los criterios para acceder a la ciudadanía y a los derechos y las oportunidades que deben reservarse a los ciudadanos. Esta situación estaría apuntando a una transformación de la ciudadanía y la soberanía unitarias en una «ciudadanía flexible» y una «soberanía dispersa». Puesto que la protección de los derechos humanos ha estado tradicionalmente en manos del Estado nacional, un Estado cuya legitimidad depende cada vez menos de una identidad cultural homogénea que marca los límites entre nosotros y los otros, es preciso «repensar la pertenencia», de tal manera que ésta no quede necesariamente ligada a la comunidad cultural, sino que atienda a la porosidad de las fronteras y a los procesos de redefinición de las identidades que tienen lugar por el contacto mutuo en un contexto de creciente movilidad transnacional. En opinión de la autora y siguiendo la tradición republicana, la ciudadanía no debería reconocerse según criterios pasivos, adscriptivos, como nacer en un territorio o un grupo étnico concreto, que son fruto del azar. Lo que debería determinar la pertenencia es lo que las personas hacen y no lo que son, su participación en los distintos ámbitos de la sociedad civil.

Creo que hay una frase de la autora que expresa muy bien cuál es el hilo conductor de su argumento y que encierra su principal aportación al debate sobre Las reivindicaciones de la cultura, y es la siguiente: «La política de la identidad y la política de la diferencia se ven afectadas por la paradoja de querer preservar la pureza de lo impuro, la inmutabilidad de lo histórico y el carácter fundamental de lo contingente» (Benhabib, 2006: 37). El conflicto, la controversia, el diálogo es algo intrínseco a 
cada una de las culturas. Sólo desde esta consideración cabe abordar una política adecuada de integración de las distintas voces que se dejan oír en una sociedad.

\section{Referencias}

SMITH, A. (1979): Nationalism in the Twentieth Century, Oxford, Oxford University Press.

TULLY, J. (1995): Strange multiplicity: constitutionalism in an age of diversity, Cambridge, University Press.

Carmen INNERARITY GRAU

\section{Jesús Casquete}

El poder de la calle. Ensayos sobre acción colectiva

(Madrid, Centro de Estudios Políticos

y Constitucionales, 2006)

No tengo miedo de tu Yavéh,

Tampoco de tu Allah,

Ni de tu Jesús,

Tengo miedo de lo que tú haces,

En el nombre de tu Dios.

Holly Near

Leyendo periódicos, visionando televisión, caminando por las calles, escuchando un mitin político, asistiendo a un partido de fútbol o a una corrida de toros, somos testigos o tenemos noticia de acciones sociales parecidas a éstas: frente a una taquilla de teatro, una audiencia tiende simultáneamente a llorar, a reír y a aplaudir; gran cantidad de gente aplaude y vitorea un gol en un partido de fútbol, mientras grupos de fans agitan sus banderas y estandartes; trabajadores en asamblea deciden arrojar sus herramientas; relacionado con los primeros rumores sobre incremento en el precio del combustible, las estaciones de servicio son visitadas por largas colas de conductores; un grupo de terroristas lleva a cabo una serie de voladuras como parte de una amplia campaña de reivindicaciones políticas de minorías étnicas; determinados grupos realizan una demostración como parte de un mitin contra el despliegue de misiles nucleares; siguiendo el ejemplo del éxito de un grupo rock, jóvenes adolescentes exhiben en sus cabellos colores vivos y la experiencia se extiende rápidamente a otras partes del país. Todos estos fenómenos, típicos de las sociedades complejas, aparecen como conductas colectivas, como conductas de masas o como movimientos sociales.

La acción colectiva es un ámbito central de la sociología. En los análisis tradicionales de la acción colectiva encontramos dos énfasis diferentes. El primero de ellos acentúa los simples hechos de la acción colectiva que aparecen como «acción sin actores», como una suma accidental de eventos individuales. Así se manifiestan los análisis de la conducta de masas (Le Bon, Tarde, Freud), para la que los motivos que surgen en el seno de la acción colectiva son el producto de una muchedumbre irracional. De la misma forma se manifiesta la tradición freudiana que enfatiza la imitación, la irracionalidad, el contagio y la sugestión. La acción social se plantea como una reacción a una 